\title{
In silico investigation of possible caffeine interactions with common inflammation-related targets
}

Lincon Fernandes de Lima Neto ${ }^{1}$, Ana Carolina Carnio Barruffini ${ }^{1}$, Douglas Vieira Thomaz ${ }^{2 *}$, Fábio Bahls Machado ${ }^{2}$, Isaac Yves Lopes de Macedo ${ }^{2}$

'PUC-GO, Goiânia, Brazil

${ }^{2}$ UFG-GO, Goiânia, Brazil

\section{ARTICLE INFO \\ Article history: \\ Received on: February 07, 2019 \\ Accepted on: March 27, 2019 \\ Available online: September 10, 2019}

Key words:

methylxanthine, molecular

modeling, inflammation,

chemoinformatics, therapeutics.

\begin{abstract}
Caffeine (CA) is a methylxanthine alkaloid widely used in anti-inflammatory drug associations due to its vasoconstricting properties. Although CA is acknowledged to interact with a plethora of macromolecules in human organism, there was to the best of our knowledge, no survey regarding its possible interactions with common inflammation-related targets. Henceforth, this work was concerned in the investigation of CA possible interactions with cyclooxygenases- 1 and -2 (COX-1 and COX-2), as well as prostaglandin $\mathrm{H} 2$ synthase- 1 and leukotriene A4 hydrolase through in silico approaches. CA molecule was studied as a ligand whereas the ligand-macromolecules docking models were created through AutoDock Vina tools. Results showcased that, although the thermodynamic features of the best scoring models did not render enough information to affirm stable interaction between CA and the analyzed macromolecules, more studies are needed to shed light on the possible role of methylxanthines towards inflammation targets.
\end{abstract}

\section{INTRODUCTION}

Caffeine (CA) is a methylxanthine alkaloid whose stimulating activities find numerous uses in human therapeutics. This compound is usually associated with non-steroidal anti-inflammatory drugs due to its vasoconstricting properties, which are promoted through CA antagonistic features over diverse cellular receptors. Nonetheless, the methylxanthine moiety (Fig. 1A and B) present in $C A$ allows its binding with adenosine receptors $\left(A_{1}, A_{2 A}, A_{2 B}\right.$, and $\mathrm{A}_{3}$ ) due to structural similarities with the physiological ligand. However, this molecule is known to also bind antagonistically with inositol triphosphate, glycine, ryanodine, and other receptors, which outlines the variety of targets prone to bound with CA [1-4].

Although literature reports concerning CA binding to the aforementioned receptors are numerous, there is a significant lack of reports regarding CA binding studies with inflammatoryresponse targets, which evidences the importance of further

*Corresponding Author

Douglas Vieira Thomaz, UFG-GO, Goiânia, Brazil.

E-mail:douglasvthomaz@gmail.com investigating this molecule in order to elucidate its therapeutic potential [5-10].

Considering studies regarding ligand-receptor binding kinetics and thermodynamics, in silico approaches offer a cheap and valuable alternative for preliminary screenings concerning chemical compounds bioactivity, moreover, these methods are also heavily used for drug discovery. In this context, molecules such as CA might be freely studied under computational strategies without the need of in vivo or in vitro assays in the first investigational steps. Nonetheless, semi-empirical approaches, such as molecular mechanics, are valuable in silico tools, which further increase their appeal in docking analysis. Under this light, the assessment of a small molecule proneness to interact with selected receptors is easily feasible by docking studies and has low computational cost [11-13].

Considering the importance of better understanding ligand-receptor features and its implications on the therapeutic applicability of small molecules, this report is aimed to explore CA interaction kinetics and thermodynamics toward the most common targets related to inflammatory response in humans. Henceforth, in silico methods based on semi-empirical approaches were used 
to investigate $\mathrm{CA}$ interaction to cyclooxygenase $(\mathrm{COX})$ isoforms (COX-1 and COX-2), as well as prostaglandin $\mathrm{H} 2$ synthase- 1 and leukotriene A4 hydrolase.

\section{EXPERIMENTAL}

\subsection{In Silico Methods}

CA (1,3,7-trimethylpurine-2,6-dione) structure was minimized through the software Chimera version 1.13 coupled to Molecular Modeling Toolkit and AMBER toolkit 4.0 prior docking studies. The same software was used to edit protein units retrieved from Protein DataBank (PDB). Moreover, the software Python Molecular Viewer version 1.5.6 was used to evaluate torsion-prone regions in CA molecule, and the docking models were conducted using AutoDock Vina and AutoDock Tools version 1.5.6. The docking model herein employed is based on a flexible ligand and a rigid receptor, therefore configuring itself in a semi-flexible model $[14,15]$.

\subsection{COXs Structures}

Human COX-1 (PDB entry: 3N8X), COX-2 (PDB entry: 5F19), prostaglandin $\mathrm{H} 2$ synthase-1 (PDB entry: $1 \mathrm{CQE}$ ), and leukotriene A4 hydrolase (PDB entry: 3FTS) were used in this study.

\section{RESULTS AND DISCUSSION}

\subsection{CA and COX-1 Interaction}

In order to explore possible $\mathrm{CA}$ and $\mathrm{COX}-1$ interactions, a docking study was performed. Figure 2 evidence the highest scoring model.

Table 1: Table of thermodynamical properties calculated for the lowest energy conformation in the docking of CA-1CQE, CA-3FTS, CA-3N8X, and CA-5F19.

\begin{tabular}{|c|c|c|c|c|}
\hline Receptor & $\begin{array}{c}\text { Affinity } \\
\left(\text { kcal.mol }^{-1}\right)\end{array}$ & $\begin{array}{c}\mathrm{Ki} \\
\left(\text { cal. } \mathrm{mol}^{-1} \cdot \mathrm{K}^{-1}\right) 10^{-5}\end{array}$ & $\begin{array}{l}\text { HBonds } \\
\text { (ligand) }\end{array}$ & $\begin{array}{l}\text { HBonds } \\
\text { (receptor) }\end{array}$ \\
\hline $1 \mathrm{CQE}$ & -6.1 & 3.60 & 1 & 1 \\
\hline 3FTS & -5.9 & 5.03 & 1 & 1 \\
\hline $3 N 8 X$ & -5.7 & 7.04 & 0 & 0 \\
\hline $5 \mathrm{~F} 19$ & -5.1 & 19.26 & 0 & 0 \\
\hline
\end{tabular}
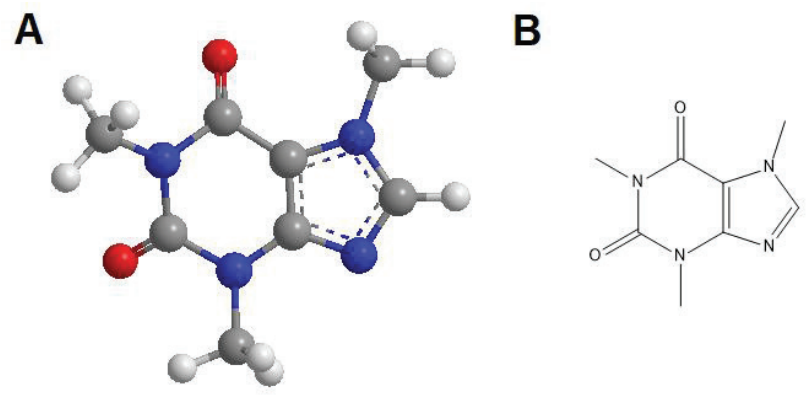

Figure 1: (A) Tri-dimensional representation of CA chemical structure. (B) CA chemical structure. Data processed in Chem3D Pro ${ }^{\circledR}$ software.
Results evidenced that CA highest scoring docking model presented no hydrogen bonds; however, the above than narrow distances found between $\mathrm{CA}$ and COX-1 electron-donor and electron-accepting moieties suggest possible intermolecular interaction [16-20].

\subsection{CA and COX-2 Interaction}

Concerning possible CA and COX-2 interactions, Figure 3 depicts the highest scoring model.

Regarding COX-2, CA highest scoring model did not present any hydrogen bonds (Figure 3). Although hydrogen bonds are nonetheless responsible for stable interactions between molecules, as well as intramolecular cohesion, their presence alone is not an indication of effective docking. In this context, other aspects such as torsional energies related to electronegative moieties, as well as molecular packaging, steric hindrance, and thermodynamic unbalance may turn hydrogen bond-rich models unfeasible. Henceforth, the first model presented highest score despite no strong bonds being detected [21-24].

\subsection{CA and Prostaglandin $\mathrm{H} 2$ Synthase-1 Interaction}

CA possible interaction with prostaglandin $\mathrm{H} 2$ synthase-1 had its highest scoring model presented in Figure 4.

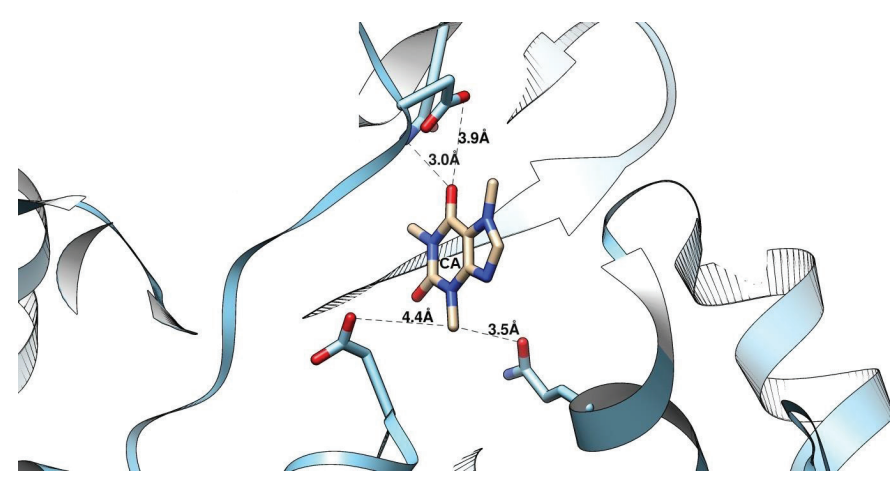

Figure 2: Docking depiction of the highest scoring model for CA-COX-1 interaction. All data gathered through Chimera software version 1.13.

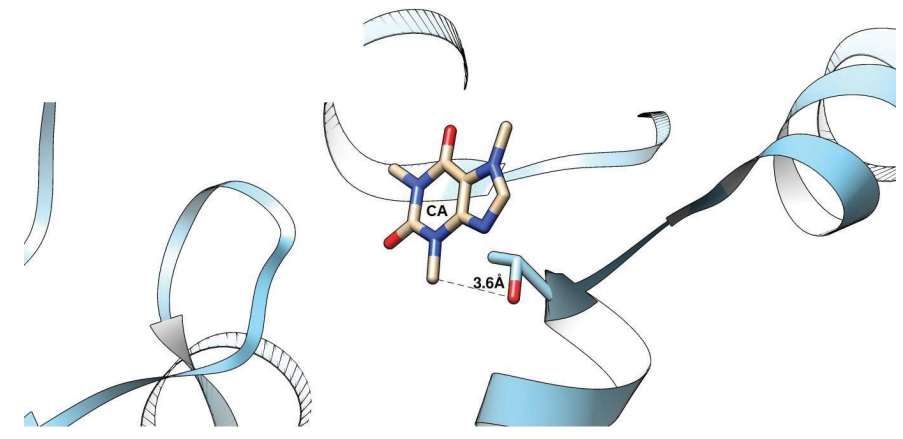

Figure 3: Docking depiction of the highest scoring model for CA-COX-2 interaction. All data gathered through Chimera software version 1.13. 
Prostaglandin $\mathrm{H} 2$ synthase-1 and CA highest scoring model evidenced that two ligand sites are hydrogen bond donors to one acceptor site in the macromolecule (Fig. 4). This result is remarkable since the energy values for the depicted model when

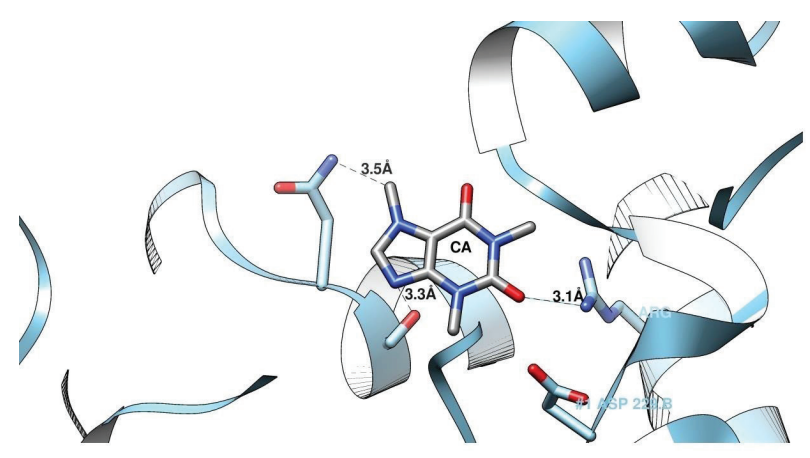

Figure 4: Docking depiction of the highest scoring model for CAProstaglandin $\mathrm{H} 2$ synthase-1 interaction. All data gathered through Chimera software version 1.13.

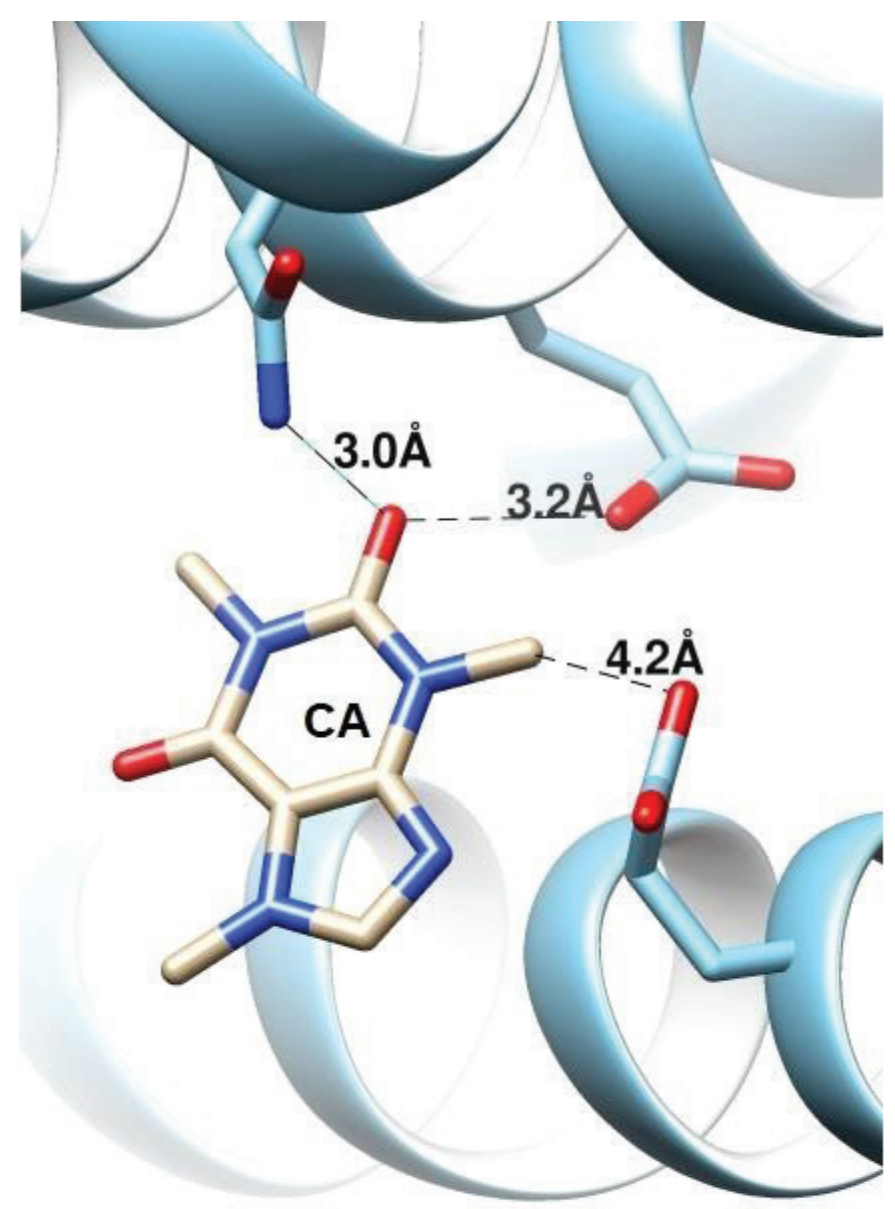

Figure 5: Docking depiction of the highest scoring model for CA-Leukotriene A4 hydrolase interaction. All data gathered through Chimera software version 1.13 associated to hydrogen bond multiplicity imply nonetheless thermodynamic feasibility of ligand-macromolecule interaction. To the best of our knowledge, there was no report concerning the possible interaction of CA and prostaglandin H2 synthase-1, henceforth, the data herein depicted, although premature, may direct further investigations [21-24].

\subsection{CA and Leukotriene $\mathrm{A} 4 \mathrm{Hydrolase}$ Interaction}

The possible interaction of CA and leukotriene A4 hydrolase was also investigated. Figure 5 depicts the highest scoring model.

Results evidenced that leukotriene A4 hydrolase and CA may possibly interact through 3 hydrogen bond donor sites in the ligand and 2 receptor sites in the macromolecule (Figure 5). Results herein depicted are nonetheless relevant, since to the best of our knowledge, no similar study concerning CA docking models with the aforementioned receptors was performed. Moreover, the amount of hydrogen bonds associated to the thermodynamic feasibility of docking may imply possible interaction between CA and leukotriene A4 hydrolase. Furthermore, other reports evidence the relevance of hydrogen bonds abundance to successful models [16-20].

\subsection{Interaction Constants}

Gibbs free energy values give insights about the proneness of an interaction between ligand-receptor when comparing different receptors to a common ligand. Thermodynamics postulates that an interaction constant is directly linked to the interactions affinity through the following equation:

$$
\Delta G=R T \ln K b
$$

Where $\Delta G$ is the interaction affinity, $R$ is the gas constant, and $T$ is the temperature. This equation can be derived in order to yield the interaction constant $K i$.

$$
K i=e^{\frac{\Delta G}{R T}}
$$

Where $e$ is the Euler's number.

When considering $\Delta \mathrm{G}$ values in molecular docking poses, higher scoring models possess smaller values of this parameter, while their $\mathrm{Ki}$ follow the same trend. In this sense, $\mathrm{Ki}$ values did corroborate to $\Delta \mathrm{G}$ in the calculated models. However, since molecular docking is prone to many false positives, more studies are needed to further investigate the findings of this work.

\section{CONCLUSION}

The present work reported an investigation of CA potential interaction with macromolecules usually involved in inflammation. Although the thermodynamic features of the best scoring models did not render enough information to affirm stable interaction between $\mathrm{CA}$ and the analyzed macromolecules, more studies are needed to shed light on the possible role of methylxanthines towards inflammation targets.

\section{CONFLICT OF INTEREST}

Authors declare no conflict of interest. 


\section{REFERENCES}

1. Esmaili Z, Heydari A. Effect of acute caffeine administration on PTZinduced seizure threshold in mice: Involvement of adenosine receptors and NO-cGMP signaling pathway. Epilepsy Res 2019;149:1-8.

2. Grant SS, Magruder KP, Friedman BH. Controlling for caffeine in cardiovascular research: a critical review. Int J Psychophysiol 2018;133:193-201.

3. Tej GNVC, Nayak PK. Mechanistic considerations in chemotherapeutic activity of caffeine. Biomed Pharmacother 2018;105:312-9.

4. Tsunoda K, Sato A, Kurata R, Mizuyama R, Shimegi S. Caffeine improves contrast sensitivity of freely moving rats. Physiol Behav 2019;199:111-7.

5. Iris M, Tsou PS, Sawalha AH. Caffeine inhibits STAT1 signaling and downregulates inflammatory pathways involved in autoimmunity. Clin Immunol 2018;192:68-77.

6. Laskar AA, Alam MF, Ahmad M, Younus H. Kinetic and biophysical investigation of the inhibitory effect of caffeine on human salivary aldehyde dehydrogenase: Implications in oral health and chemotherapy. J Mol Struct 2018;1157:61-8.

7. Padbury JF. Caffeine, inflammation, and BPD. J Pediatr 2011;158(1):A1.

8. Reef TA, Ghanem E. Caffeine: well-known as psychotropic substance, but little as immunomodulator. Immunobiology 2018;223(12):818-25.

9. Sayin K, Üngördü A. Investigation of anticancer properties of caffeinated complexes via computational chemistry methods. Spectrochim Acta Part A Mol Biomol Spectroscopy 2018;193:147-55.

10. Wang W, Zhang W, Duan Y, Jiang Y, Zhang L, Zhao B, et al. Investigation of the binding sites and orientation of caffeine on human serum albumin by surface-enhanced Raman scattering and molecular docking. Spectrochim Acta Part A Mol Biomol Spectroscopy 2013;115:57-63.

11. Amaro RE, Baudry J, Chodera J, Demir O, McCammon JA, Miao Y, et al. Ensemble docking in drug discovery. Biophys J 2018;114(10):2271-8.

12. García-Nieto J, López-Camacho E, García-Godoy MJ, Nebro AJ, Aldana-Montes JF. Multi-objective ligand-protein docking with particle swarm optimizers. Swarm and Evol Comput 2019;44:439-52.

13. Gupta M, Sharma R, Kumar A. Docking techniques in pharmacology: How much promising? Comput Biol Chem 2018;76:210-7.

14. Salomon-Ferrer R, Case DA, Walker RC. An overview of the Amber biomolecular simulation package. WIRES Comput Mol Sci 2013;3:198-210.
15. Case DA, Cheatham TE, Darden IT, Gohlke H, Luo R, Merz KM, et al. The Amber biomolecular simulation programs. J Comput Chem 2005;26:1668-88.

16. Jiang X, Tsona NT, Tang S, Du L. Hydrogen bond docking preference in furans: $\mathrm{OH}^{\cdots} \pi$ vs. $\mathrm{OH}^{\cdots} \mathrm{O}$. Spectrochim Acta Part A Mol Biomol Spectroscopy 2018;191:155-64.

17. Khorasani R, Fleming PE. On calculating HR bond enthalpies using computational data. Comput Theor Chem 2016;1096:89-93.

18. Kumar SP. PLHINT: A knowledge-driven computational approach based on the intermolecular $\mathrm{H}$ bond interactions at the protein-ligand interface from docking solutions. J Mol Graph Model 2018;79: 194-212.

19. Lynch DE, Reeves CR. Statistical analysis of the effect of a single $\mathrm{OH}$ hydrogen-bonding interaction on carbonyl bond lengths. J Mol Structure 2019;1180:158-62.

20. Zhao H, Tang S, Du L. Hydrogen bond docking site competition in methyl esters. Spectrochim Acta Part A Mol Biomol Spectroscopy 2017;181:122-30.

21. Cosconati S, Forli S, Perryman AL, Harris R, Goodsell DS, Olson AJ. Virtual screening with AutoDock: theory and practice. Expert Opin Drug Discov 2010;5(6):597-607.

22. Meng X, Zhang H, Mezei M, Cui M. Molecular Docking: a powerful approach for structure-based drug discovery. Curr Comput Aided Drug Design 2011;7(2):146-57.

23. Morris GM, Lim-Wilby M. Molecular docking. Methods Mol Biol 2008;443:365-82.

24. Wu MY, Dai DQ, Yan H. PRL-Dock: protein-ligand docking based on hydrogen bond matching and probabilistic relaxation labeling. Proteins 2012;80(9):2137-53.

How to cite this article:

de Lima Neto LF, Barruffini ACC, Thomaz DV, Machado FB, Macedo IYL. In silico investigation of possible Caffeine interactions with common Inflammation-related targets. J Appl Biol Biotech 2019;7(05):31-34. 